\title{
A Game Theoretic Framework for Distributed Control of Multi-Agent Systems with Acyclic Communication Topologies
}

\author{
Domenico Cappello and Thulasi Mylvaganam
}

\begin{abstract}
A multi-agent system consisting of heterogeneous agents, described by nonlinear dynamics and with inter-agent communication characterised by a directed acyclic graph, is considered in this paper. A framework for designing distributed control strategies obtained via the combination of local non-cooperative differential games is provided. The resulting dynamic (local) state-feedback control laws can be computed offline and in a decentralised manner. Conditions for ensuring stability of the overall closed-loop system are provided, before the proposed game theoretic framework is applied to a formation control problem.
\end{abstract}

\section{INTRODUCTION}

The technological advances of the last decades have resulted in a growing interest in solving complex engineering tasks in a distributed fashion [1]. Multiagent systems (MAS), in particular, have proved to be useful for a wide range of applications [2]. A MAS is a system composed of multiple autonomous agents, entities usually characterised by limited processing and computation capabilities, each taking decisions based on its individual objective. Agents in these systems are often required to operate together in order to achieve a global objective. Common examples of MAS are multirobot systems [3]-[7], sensor networks [8], power systems [9], [10], and radio transmission systems for cell phone networks [11]. Since classical centralised control techniques are not practically applicable to many of these complex large-scale systems, distributed control techniques are required. Many different approaches for designing distributed control laws can be found in the literature (see e.g. [12]-[14] and references therein). Some of these approaches are based on differential game theory [15]-[19], which studies the strategic interactions of individual decision-making players. These players affect, through the selection of their strategies, the behaviour of a common dynamical system. While classical differential games require "centralised reasoning", interesting results in the direction of distributed control are emerging. E.g. in [20] graphical games for dynamical systems are defined, and convergence properties of the policy iteration algorithm are investigated.

In this paper we consider a MAS with agents described by input-affine, nonlinear dynamics subject to

D. Cappello is with the Department of Aeronautics, Imperial College London, London SW7 2AZ, UK (Email: domenico.cappello16@imperial.ac.uk).

T. Mylvaganam is with the Department of Aeronautics, Imperial College London, London SW7 2AZ, UK (Email: t.mylvaganam@imperial.ac.uk). limited communication. We define a modular, decentralised game-theoretic framework which guarantees, under given assumptions, asymptotic stability of the zero-equilibrium of the global system. The main contributions of this paper are twofold. First, we exploit notions from game theory to formulate a distributed differential game as a combination of local differential games. Second, we provide a constructive approach to solve this distributed game and provide conditions to ensure stability properties for the overall system. Differently from [17]-[20], the approach is applicable to nonlinear systems and does not rely on the agents sharing information regarding their strategies. Differently from [20], the distributed control strategies are computed offline as the solution of (fictitious) noncooperative local differential games which reflect the incomplete and possibly erroneous information each agent has of its neighbourhood. The remainder of this paper is organised as follows. In Section II a MAS consisting of heterogeneous agents is defined and local overlapping subsystem dynamics, based on the considered time-invariant inter-agent communication topology, are specified. In Section III local and distributed differential games are defined, together with the notion of $\epsilon_{\alpha}$-Nash equilibria. Solutions of the local and distributed differential games are then provided in Section IV. In Section V the obtained theoretical results are illustrated by means of an example concerning distributed formation control. Finally, some concluding remarks are given in Section VI.

Notation. $\mathbb{R}$ denotes the set of real numbers, $\mathbb{C}^{-}$denotes the set of complex numbers with negative real part. The $n \times n$ identity matrix is denoted by $I_{n}$, the zero matrix is denoted by 0 and $\sigma(A)$ denotes the spectrum of a square matrix $A$. blockdiag $\left(A_{1}, \ldots, A_{N}\right)$ denotes a block diagonal matrix with diagonal blocks $A_{1}, \ldots, A_{N}$. The cardinality of a set $\mathcal{S}$ is denoted as $|\mathcal{S}|$ and $\sum_{j \in \mathcal{S}} y_{j}$ denotes the summation of all $y_{j}$ such that $j \in \mathcal{S}$. The gradient of a function $V: \mathbb{R}^{n} \rightarrow \mathbb{R}$ is denoted by $\frac{\partial V}{\partial x}$.

\section{PRELIMINARIES}

We consider a MAS composed of $N$ heterogeneous agents characterised by (possibly) different dynamics and individual objectives. Communication between agents is characterised by a directed graph $\mathcal{G}(\mathcal{V}, \mathcal{E})$, where the set of nodes $\mathcal{V}$ represents the agents and the set of edges $\mathcal{E}$ represents the information flow. 
Namely, given $i \in \mathcal{V}$ and $j \in \mathcal{V},(i, j) \in \mathcal{E}$ implies that agent $i$ shares its information with agent $j$. We use the convention that $(i, i) \notin \mathcal{E}$, for all $i \in \mathcal{V}$.

Assumption 1: The inter-agent communication graph $\mathcal{G}(\mathcal{V}, \mathcal{E})$ is a static directed acyclic graph, and the set of nodes $\mathcal{V}$ is ordered according to a topological sorting ${ }^{1}$ of $\mathcal{G}(\mathcal{V}, \mathcal{E})$.

For each agent $i$ in the MAS, we introduce two sets: the set of (1-hop) neighbours ${ }^{2} \mathcal{N}_{i}$ defined as the set of all agents $j$ such that $(j, i) \in \mathcal{E}$ as well as agent $i$ itself, namely $\mathcal{N}_{i}=\{j:(j, i) \in \mathcal{E}\} \cup\{i\}$, and the set of 2-hop neighbours $\mathcal{N}_{i}^{2}=\left\{k:(k, j) \in \mathcal{E}\right.$, for some $\left.j \in \mathcal{N}_{i}\right\} \backslash \mathcal{N}_{i}$.

We consider agents described by dynamics of the form

$$
\dot{x}_{i}=f_{i}\left(x_{i}\right)+\sum_{j \in \mathcal{N}_{i}} g_{i j}\left(x_{i}\right) u_{j},
$$

where $f_{i}: \mathbb{R}^{n_{i}} \rightarrow \mathbb{R}^{n_{i}}$ and $g_{i j}: \mathbb{R}^{n_{i}} \rightarrow \mathbb{R}^{n_{i} \times p_{j}}$ are smooth mappings, $x_{i} \in \mathbb{R}^{n_{i}}$ is the state of the $i$-th agent and $u_{j} \in \mathbb{R}^{p_{j}}$ is the control input of the $j$-th agent, for $j \in \mathcal{N}_{i}, i=1, \ldots, N$. We refer to the system (1) as the individual system of agent $i$, and assume that the origin of system (1), with $u_{i}=0$, is an equilibrium, i.e. $f_{i}(0)=0$. This assumption implies the existence of a matrix-valued mapping $F_{i}: \mathbb{R}_{i}^{n} \rightarrow \mathbb{R}_{i}^{n} \times \mathbb{R}_{i}^{n}$ such that $f_{i}\left(x_{i}\right)=F_{i}\left(x_{i}\right) x_{i}$.

Let $x=\left[x_{1}^{\top}, \ldots, x_{N}^{\top}\right]^{\top} \in \mathbb{R}^{n}$ denote the state of the global system. The global system satisfies the dynamics

$$
\dot{x}=F(x) x+\sum_{j=1}^{N} G_{j}(x) u_{j},
$$

where $F(x)=\operatorname{blockdiag}\left(F_{1}, \ldots, F_{N}\right), G_{j}(x)=$ $\left[g_{1 j}^{\top}, \ldots, g_{N j}^{\top}\right]^{\top}$, for $i=1, \ldots, N$, and $n \triangleq \sum_{i=1}^{N} n_{i}$.

We consider the case in which every agent $i \in \mathcal{V}$ has continuous access to the states $x_{j} \in \mathcal{N}_{i}$ and has knowledge of the functions $f_{j}$ and $g_{j k}, j \in \mathcal{N}_{i}, k \in \mathcal{N}_{i}$. The collection of this information forms the local state of agent $i$, defined as

$$
\hat{x}_{i} \triangleq N_{i} x=\left[x_{i}^{\top}, x_{j_{2}}^{\top}, \cdots, x_{\left.j_{\left|\mathcal{N}_{i}\right|}\right]^{\top}}^{\top},\right.
$$

where $\hat{x}_{i} \in \mathbb{R}^{\hat{n}_{i}}, N_{i} \in \mathbb{R}^{\hat{n}_{i} \times n}, \hat{n}_{i}=\sum_{j \in \mathcal{N}_{i}} n_{j}$, and $i, j_{2}, \ldots, j_{\left|\mathcal{N}_{i}\right|} \in \mathcal{N}_{i}$. The local state (3) evolves according to the true local dynamics

$$
\dot{\hat{x}}_{i}=\hat{F}_{i}\left(\hat{x}_{i}\right) \hat{x}_{i}+\sum_{j \in \mathcal{N}_{i}} \hat{g}_{i j}\left(\hat{x}_{i}\right) u_{j}+\sum_{j \in \mathcal{N}_{i}^{2}} \hat{g}_{i j}\left(\hat{x}_{i}\right) u_{j},
$$

where $\hat{F}_{i}\left(\hat{x}_{i}\right)=\operatorname{blockdiag}\left(F_{i}\left(x_{i}\right), F_{j_{2}}\left(x_{j_{2}}\right), \ldots\right.$, $F_{j_{\left|\mathcal{N}_{i}\right|}}\left(x_{\left.j_{\left|\mathcal{N}_{i}\right|}\right)}\right)$, for $i, j_{2}, \ldots, j_{\left|\mathcal{N}_{i}\right|} \in \mathcal{N}_{i}$, and $\hat{g}_{i j}=N_{i} G_{j}(x)$, for $j \in \mathcal{N}_{i} \cup \mathcal{N}_{i}^{2}$. Note that, since agent $j$ does not share information regarding its

\footnotetext{
${ }^{1} \mathrm{~A}$ topological sorting of a directed acyclic graph $\mathcal{G}(\mathcal{V}, \mathcal{E})$ is a complete ordering of the vertices in $\mathcal{V}$ such that, for every directed edge $(u, v) \in \mathcal{E}, u$ comes before $v$ in the ordering.

${ }^{2}$ Given a directed graph $\mathcal{G}(\mathcal{V}, \mathcal{E}), u \in \mathcal{V}$ is said to be a $k$-hop neighbour of $v \in \mathcal{V}$ if the length of the shortest path from $u$ to $v$ is $k$.
}

strategy with agent $i, i \neq j$, the true local dynamics is not known to agent $i$.

The main contribution of this paper is a systematic procedure to design distributed local state-feedback control laws which render the zero equilibrium of the global system (2) locally asymptotically stable. The procedure relies on the concepts of local differential games and distributed differential games, introduced in the following section.

\section{Distributed DifFERENTIAL GAMES}

We consider the case in which each agent seeks to achieve an individual objective described by a cost functional to be minimised. The cost functional associated with each agent $i, i=1, \ldots, N$, depends on its local state (3) and, differently from "classical" differential games (see e.g. [21]), since the true local dynamics (4) is unknown to agent $i$, it can only depend on some assumed evolution of the local state inferred from the information available to the agent. More precisely, agent $i$ does not know the control strategies of its 2-hop neighbours, and it may have some (incomplete) information regarding the control strategies of its neighbours.

Suppose agent $i$ assumes that its neighbours adopt the strategies $\bar{u}_{i j}(\cdot): \mathbb{R} \rightarrow \mathbb{R}^{p_{i}}$, for $j \in \mathcal{N}_{i}$, with $\bar{u}_{i i}=u_{i}$, for $i=1, \ldots, N$, and let $\overline{\mathbf{u}}_{\mathbf{i} \mathbf{j}_{\mathrm{k}}}$ denote the set of strategies $\bar{u}_{i j}$, for $j \in \mathcal{N}_{i} \backslash\left\{j_{k}\right\}$. Based on its partial knowledge of its local system and these assumptions, agent $i$ infers that its local state evolves according to its assumed local dynamics

$$
\dot{\bar{x}}_{i}=\hat{F}_{i}\left(\bar{x}_{i}\right) \bar{x}_{i}+\sum_{j \in \mathcal{N}_{i}} \hat{g}_{i j}\left(\bar{x}_{i}\right) \bar{u}_{i j}
$$

with $\bar{x}_{i}(0)=\hat{x}_{i}(0)$, where $\bar{x}_{i}(\cdot): \mathbb{R} \rightarrow \mathbb{R}^{\hat{n}_{i}}$ is an "estimate" of the local state of agent $i$. Namely, $\bar{x}_{i}$ represents the assumed evolution of the local state (whereas $\hat{x}_{i}$ described by (4) represents its actual evolution).

Agent $i$ seeks to minimise (with respect to $u_{i}$ ) a cost functional under the assumption that its neighbours seek to minimise their own objectives, subject to the assumed local dynamics of agent $i$. That is, agent $i$ solves a so-called local differential game defined by cost functionals

$$
J_{i j}\left(\bar{x}_{i}(0), \bar{u}_{i j}, \overline{\mathbf{u}}_{\mathbf{i j}}\right)=\frac{1}{2} \int_{0}^{\infty}\left(q_{i j}\left(\bar{x}_{i}\right)+\bar{u}_{i j}^{\top} R_{j j} \bar{u}_{i j}\right) d t
$$

for all $j \in \mathcal{N}_{i}$, subject to the assumed dynamics (5), wherein $q_{i j}\left(\bar{x}_{i}\right) \triangleq \bar{x}_{i}^{\top} Q_{i j}\left(\bar{x}_{i}\right) \bar{x}_{i}$ and $g_{i j}\left(\bar{u}_{i j}\right)=\bar{u}_{i j}^{\top} R_{j j} \bar{u}_{i j}$ represent a running cost and a control penalty, respectively, for $j \in \mathcal{N}_{i}$.

Assumption 2: The running costs $q_{i j}\left(\bar{x}_{i}\right)$, for $j \in \mathcal{N}_{i}$, are such that $\sum_{j \in \mathcal{N}_{i}} Q_{i j}\left(\bar{x}_{i}\right)>0$, for all $\bar{x}_{i} \in \mathbb{R}^{\hat{n}_{i}}$, for $i=1, \ldots, N$.

Remark 1: The selection of $Q_{i j}\left(\bar{x}_{i}\right)$ could be arbitrary to reflect any information available to agent $i$. However, its choice strongly influences the performance of the 
strategy of agent $i$ in terms of its objective. For instance, one choice of $Q_{i j}\left(\bar{x}_{i}\right)$ could be a function of $Q_{j j}\left(\bar{x}_{j}\right)$ that only depends on $x_{k}$, for $k \in \mathcal{N}_{i} \cap \mathcal{N}_{j}$, e.g. $q_{i j}\left(\bar{x}_{i}\right)$ equals to $q_{j j}\left(\bar{x}_{j}\right)$ calculated in $x_{l}=0$, for $l \notin \mathcal{N}_{i} \cap \mathcal{N}_{j}$. This setting is similar to the one considered in [19] and is such that, in the full information case, the "classical" differential game, i.e. the differential game defined by cost functionals (6), subject to the dynamics of the global system (2), is recovered.

A solution of the local game is given in terms of assumed admissible strategies defined as follows.

Definition 1: A set of assumed feedback strategies $\left\{\bar{u}_{i j_{1}}, \ldots, \bar{u}_{\left.i j_{\left|\mathcal{N}_{i}\right|}\right\}}\right\}, j_{1}, \ldots, j_{\left|\mathcal{N}_{i}\right|} \in \mathcal{N}_{i}$, is admissible if the zero-equilibrium of the assumed local system (5) in closed-loop with $\bar{u}_{i j_{1}}, \ldots, \bar{u}_{i j_{\left|\mathcal{N}_{i}\right|}}$, with $j_{1}, \ldots, j_{\left|\mathcal{N}_{i}\right|} \in \mathcal{N}_{i}$, is locally asymptotically stable.

Problem 1: Considering agent $i$ and its set of neighbours, determine a feedback Nash equilibrium of the nonzero-sum differential game defined by the cost functionals (6), $j \in \mathcal{N}_{i}$, subject to the assumed dynamics (5), i.e. find a set of assumed admissible feedback strategies $\left\{\bar{u}_{i j_{1}}^{*}, \ldots, \bar{u}_{i j_{\left|\mathcal{N}_{i}\right|} \mid}^{*}\right\}, j_{1}, \ldots, j_{\left|\mathcal{N}_{i}\right|} \in \mathcal{N}_{i}$, such that $J_{i j}\left(\bar{x}_{i}(0), \bar{u}_{i j}^{*}, \overline{\mathbf{u}}_{\mathbf{i j}}^{*}\right) \leq J_{i j}\left(\bar{x}_{i}(0), \bar{u}_{i j}, \overline{\mathbf{u}}_{\mathbf{i j}}^{*}\right)$, for all assumed admissible strategies $\left\{\bar{u}_{i j}, \overline{\mathbf{u}}_{\mathbf{i j}}^{*}\right\}$, for all $j \in \mathcal{N}_{i}$.

Finding a Nash equilibrium solution of the differential game defined by cost functionals (6), $j \in \mathcal{N}_{i}$, subject to dynamics (5), involves solving the Hamilton-JacobiIsaacs (HJI) partial differential equations (PDEs), which is an intractable problem. In [22], the authors introduce the notion of $\epsilon_{\alpha}$-Nash equilibrium, which represents a relaxation of a Nash equilibrium, and involves only the solution of partial differential inequalities (PDIs). In the setting considered herein, obtaining an $\epsilon_{\alpha}$-Nash equilibrium solution for the local differential game entails finding functions $V_{i j}$ satisfying the inequalities

$$
\begin{gathered}
\frac{1}{2} q_{i j}\left(\bar{x}_{i}\right)+\frac{1}{2} \frac{\partial V_{i j}}{\partial \bar{x}_{i}} \hat{g}_{i j}\left(\bar{x}_{i}\right) R_{j j}^{-1} \hat{g}_{i j}^{\top}\left(\bar{x}_{i}\right) \frac{\partial V_{i j}^{\top}}{\partial \bar{x}_{i}}+\frac{\partial V_{i j}}{\partial \bar{x}_{i}} \hat{f}_{i}\left(\bar{x}_{i}\right) \\
-\sum_{k \in \mathcal{N}_{i}} \frac{\partial V_{i j}}{\partial \bar{x}_{i}} \hat{g}_{i k}\left(\bar{x}_{i}\right) R_{k k}^{-1} \hat{g}_{i k}^{\top}\left(\bar{x}_{i}\right) \frac{\partial V_{i k}^{\top}}{\partial \bar{x}_{i}} \leq 0
\end{gathered}
$$

for $j \in \mathcal{N}_{i}$, for $i=1, \ldots, N$. Following the approach first introduced in [22], a local solution to PDIs (7) which requires only the solution of algebraic equations, can be constructed systematically using dynamic control laws of the form

$$
\bar{u}_{i j}=\beta_{i j}\left(\bar{x}_{i}, \xi_{i}\right), \quad \dot{\xi}_{i}=\tau_{i}\left(\bar{x}_{i}, \xi_{i}\right),
$$

where $\beta_{i j}: \mathbb{R}^{\hat{n}_{i}} \times \mathbb{R}^{\hat{n}_{i}} \rightarrow \mathbb{R}^{p_{i}}$ and $\tau_{i}: \mathbb{R}^{\hat{n}_{i}} \times \mathbb{R}^{\hat{n}_{i}} \rightarrow \mathbb{R}^{\hat{n}_{i}}$ are smooth mappings such that $\beta_{i j}(0,0)=0, \tau_{i}(0,0)=$ $0, \xi_{i} \in \mathbb{R}^{\hat{n}_{i}}$, for $j \in \mathcal{N}_{i}$, for $i=1, \ldots, N$. For clarity of presentation, we define admissible and $\alpha$-admissible (dynamic) control laws as follows.

Definition 2: A set of assumed dynamic feedback strategies $\left\{\bar{u}_{i j_{1}}, \ldots, \bar{u}_{\left.i j_{\left|\mathcal{N}_{i}\right|}\right\}}\right\}, j_{1}, \ldots, j_{\left|\mathcal{N}_{i}\right|} \in \mathcal{N}_{i}$, is admissible if the zero-equilibrium of the closed-loop system (5)-(8) is locally asymptotically stable.
Definition 3: A set of assumed dynamic feedback strategies $\left\{\bar{u}_{i j_{1}}, \ldots, \bar{u}_{i j_{\left|\mathcal{N}_{i}\right|}}\right\}, j_{1}, \ldots, j_{\left|\mathcal{N}_{i}\right|} \in \mathcal{N}_{i}$, is $\alpha-$ admissible if the zero-equilibrium of the closed-loop system (5)-(8) is locally exponentially stable with a local rate of convergence greater than $\alpha$, i.e. $\sigma\left(A_{c l}+\alpha I\right) \in \mathbb{C}^{-}$, where $A_{c l}$ is the matrix describing the system (5)-(8) linearised about the origin.

We can now define the notion of $\epsilon_{\alpha}$-Nash equilibrium (in terms of dynamic feedback strategies).

Problem 2: Considering agent $i$ and its set of neighbours, determine a feedback $\epsilon_{\alpha}$-Nash equilibrium of the nonzero-sum differential game defined by the cost functionals (6), $j \in \mathcal{N}_{i}$, subject to the assumed dynamics (5), i.e. find a set of assumed admissible dynamic feedback strategies $\left\{\bar{u}_{i j_{1}}^{*}, \ldots, \bar{u}_{i j_{\left|\mathcal{N}_{i}\right|}^{*}}\right\}, j_{1}, \ldots, j_{\left|\mathcal{N}_{i}\right|} \in \mathcal{N}_{i}$, such that there exists a constant $\epsilon_{X_{0}, \alpha} \geq 0$, parameterised with respect to both the initial (extended) state $\left[\bar{x}_{i}(0)^{\top}, \xi_{i}(0)^{\top}\right]^{\top}=X_{0}$, and $\alpha \geq 0$, such that

$$
J_{i j}\left(X_{0}, \bar{u}_{i j}^{*}, \overline{\mathbf{u}}_{\mathbf{i j}}^{*}\right) \leq J_{i j}\left(X_{0}, \bar{u}_{i j}, \overline{\mathbf{u}}_{\mathbf{i j}}^{*}\right)+\epsilon_{X_{0}, \alpha},
$$

for all $\alpha$-admissible dynamic feedback strategies $\left\{\bar{u}_{i j}, \overline{\mathbf{u}}_{\mathbf{i j}}^{*}\right\}$, for all $j \in \mathcal{N}_{i}$.

We refer to Problems 1 and 2 as local differential games for agent $i$. The collection of the local differential games forms a distributed differential game.

Problem 3 (Distributed differential game): Consider system (1), for $i=1, \ldots, N$, and graph $\mathcal{G}(\mathcal{V}, \mathcal{E})$ for which Assumption 1 holds. Determine $\beta_{i j}\left(\bar{x}_{i}, \xi_{i}\right)$ and $\tau_{i}\left(\bar{x}_{i}, \xi_{i}\right)$ such that the dynamic control laws (8), for $j \in \mathcal{N}_{i}$, constitute a solution of Problem 2, for $i=1, \ldots, N$, and such that the origin of the global system (2) in closed-loop with ${ }^{3} u_{i}=\beta_{i i}\left(\hat{x}_{i}, \xi_{i}\right)$, $\dot{\xi}_{i}=\tau_{i}\left(\hat{x}_{i}, \xi_{i}\right)$, for $i=1, \ldots, N$, is locally asymptotically stable.

In the following section we present a solution, which can be determined offline in a decentralised manner, to Problems 2 and 3. Note that, when exact solutions to Problem 1 are available, the distributed game can be defined in terms of Problem 1 in place of Problem 2.

\section{Solution of Distributed Differential Games}

For clarity of presentation, we construct a solution to Problem 3 in three steps, outlined in the following subsections. For each local differential game, i.e. Problem 2, the state $\bar{x}_{i} \in \mathbb{R}^{\hat{n}_{i}}$, describing the assumed evolution of the local system, together with the state $\xi_{i} \in \mathbb{R}^{\hat{n}_{i}}$ of the controller introduced in (8), form the extended state of the assumed closed-loop system (5)-(8). On this extended space we exploit the results of [22] to define functions $V_{i j}: \mathbb{R}^{\hat{n}_{i}} \times \mathbb{R}^{\hat{n}_{i}} \rightarrow \mathbb{R}$, namely $V_{i j}\left(\bar{x}_{i}, \xi_{i}\right)$, which constitute a local solution to a system of coupled PDIs

\footnotetext{
${ }^{3}$ Note that the dynamic control laws are computed in terms of the states $\hat{x}_{i}$ (which evolve according to the true local dynamics (4)), in place of the states $\bar{x}_{i}$ (which evolve according to the assumed local dynamics (5)), $i=1, \ldots, N$.
} 
(defined on the extended state $\left(\bar{x}_{i}, \xi_{i}\right)$ ), namely

$$
\begin{gathered}
\mathcal{H} \mathcal{J}_{i j}\left(\bar{x}_{i}, \xi\right) \triangleq \frac{1}{2} \frac{\partial V_{i j}}{\partial \bar{x}_{i}} \hat{g}_{i j}\left(\bar{x}_{i}\right) R_{j j}^{-1} \hat{g}_{i j}^{\top}\left(\bar{x}_{i}\right) \frac{\partial V_{i j}{ }^{\top}}{\partial \bar{x}_{i}} \\
-\sum_{k \in \mathcal{N}_{i}} \frac{\partial V_{i j}}{\partial \bar{x}_{i}} \hat{g}_{i k}\left(\bar{x}_{i}\right) R_{k k}^{-1} \hat{g}_{i k}^{\top}\left(\bar{x}_{i}\right) \frac{\partial V_{i k}{ }^{\top}}{\partial \bar{x}_{i}} \\
+\frac{1}{2} q_{i j}\left(\bar{x}_{i}\right)+\frac{\partial V_{i j}}{\partial \bar{x}_{i}} \hat{f}_{i}\left(\bar{x}_{i}\right)+\frac{\partial V_{i j}}{\partial \xi_{i}} \dot{\xi}_{i} \leq 0,
\end{gathered}
$$

for $j \in \mathcal{N}_{i}, i=1, \ldots, N$. The design of such functions relies on an important notion first introduced in [22]. This tool, which we refer to as algebraic $\bar{P}$ matrix solution, is fundamental to construct a (local) solution of Problem 2.

Definition 4 (Algebraic $\bar{P}$ Matrix Solution): Let $\Sigma_{i j}$ : $\mathbb{R}^{\hat{n}_{i}} \rightarrow \mathbb{R}^{\hat{n}_{i} \times \hat{n}_{i}}, \Sigma_{i j}\left(\bar{x}_{i}\right)=\Sigma_{i j}^{\top}\left(\bar{x}_{i}\right)>0$ for all $\bar{x}_{i} \in \chi \subseteq$ $\mathbb{R}^{\hat{n}_{i}}, 0 \in \chi$. The $\mathcal{C}^{1}$ matrix-valued functions $P_{i j}: \mathbb{R}^{\hat{n}_{i}} \rightarrow$ $\mathbb{R}^{\hat{n}_{i} \times \hat{n}_{i}}$ are said to be $\chi$-algebraic $\bar{P}$ matrix solutions of (7) provided that

(i) for all $\bar{x}_{i} \in \chi$,

$$
\begin{aligned}
& Q_{i j}\left(\bar{x}_{i}\right)+P_{i j}\left(\bar{x}_{i}\right) \hat{g}_{i j}\left(\bar{x}_{i}\right) R_{j j}^{-1} \hat{g}_{i j}^{\top}\left(\bar{x}_{i}\right) P_{i j}\left(\bar{x}_{i}\right) \\
& +\sum_{i j}\left(\bar{x}_{i}\right)+P_{i j}\left(\bar{x}_{i}\right) \hat{F}_{i}\left(\bar{x}_{i}\right)+\hat{F}_{i}^{\top}\left(\bar{x}_{i}\right) P_{i j}\left(\bar{x}_{i}\right) \\
& -\sum_{k \in \mathcal{N}_{i}} P_{i j}\left(\bar{x}_{i}\right) \hat{g}_{i k}\left(\bar{x}_{i}\right) R_{k k}^{-1} \hat{g}_{i k}^{\top}\left(\bar{x}_{i}\right) P_{i k}\left(\bar{x}_{i}\right) \\
& -\sum_{k \in \mathcal{N}_{i}} P_{i k}^{\top}\left(\bar{x}_{i}\right) \hat{g}_{i k}\left(\bar{x}_{i}\right) R_{k k}^{-1} \hat{g}_{i k}^{\top}\left(\bar{x}_{i}\right) P_{i j}\left(\bar{x}_{i}\right)=0,
\end{aligned}
$$

(ii) the mappings are such that $\sum_{i=1}^{N} P_{i i}(0)>0$,

with $P_{i j}\left(\bar{x}_{i}\right)=P_{i j}^{\top}\left(\bar{x}_{i}\right)$, for all $\bar{x}_{i} \in \chi$, for all $j \in \mathcal{N}_{i}$, $i=1, \ldots, N$. If $\chi=\mathbb{R}^{\hat{n}_{i}}, P_{i j}$ are said to be algebraic $\bar{P}$ matrix solutions of (7), for $j \in \mathcal{N}_{i}$.

\section{A. Solution of Local Differential Games}

We recall the main result of [22] (the interested reader is referred to [22, Theorem 4] for more details).

Theorem 1: Consider agent $i$ and the local differential game, given in Problem 2, defined by cost functionals (6), for $j \in \mathcal{N}_{i}$, subject to the assumed dynamics (5), and suppose that Assumption 2 holds. Let $P_{i j}$ be an algebraic $\bar{P}$ matrix solution of (7), for $j \in \mathcal{N}_{i}$, and let $S_{i j} \in \mathbb{R}^{\hat{n}_{i} \times \hat{n}_{i}}, S_{i j}=S_{i j}^{\top}>0$ be such that

$$
\sum_{k \in \mathcal{N}_{i}}\left(S_{i j} S_{i k}+S_{i k} S_{i j}\right)>0
$$

for $j \in \mathcal{N}_{i}$. Then there exist $\bar{k}_{i} \geq 0$ and a non-empty set $\Omega_{i} \subseteq \mathbb{R}^{\hat{n}_{i}} \times \mathbb{R}^{\hat{n}_{i}}, 0 \in \Omega_{i}$, such that

$$
V_{i j}\left(\bar{x}_{i}, \xi_{i}\right)=\frac{1}{2} \bar{x}_{i}^{\top} P_{i j}\left(\xi_{i}\right) \bar{x}_{i}+\frac{1}{2}\left(\bar{x}_{i}-\xi_{i}\right)^{\top} S_{i j}\left(\bar{x}_{i}-\xi_{i}\right),
$$

for $j \in \mathcal{N}_{i}$, solve the system of PDIs (10), for $j \in \mathcal{N}_{i}$, for all $\left(\bar{x}_{i}, \xi_{i}\right) \in \Omega_{i}$, with $\dot{\xi}_{i}=-k_{i} \sum_{j \in \mathcal{N}_{i}} \frac{\partial V_{i j}{ }^{\top}}{\partial \xi_{i}}$, for all $k_{i} \geq \bar{k}_{i}$. It follows that there exists a neighbourhood of the origin where the dynamic feedback strategies

$$
\dot{\xi}_{i}=-k_{i} \sum_{j \in \mathcal{N}_{i}} \frac{\partial V_{i j}{ }^{\top}}{\partial \xi_{i}}, \quad \bar{u}_{i j}^{*}=-R_{j j}^{-1} \hat{g}_{i j}^{\top}\left(\bar{x}_{i}\right) \frac{\partial V_{i j}{ }^{\top}}{\partial \bar{x}_{i}},
$$

for $j \in \mathcal{N}_{i}$, are a feedback $\epsilon_{\alpha}$-Nash equilibrium of the local differential game in $\Omega_{i}$.

While the inequalities (10), $j \in \mathcal{N}_{i}$, are satisfied for the assumed dynamics (5), differently from [22], no stability property can, in general, be expected to hold for the global closed-loop system (2)-(14), for $j=i$, for $i=1, \ldots, N$. This issue is addressed in the following subsection.

\section{B. Stability Conditions}

Suppose Assumption 1 holds and consider the system (1) in closed-loop with the dynamic controllers

$$
\begin{aligned}
\dot{\xi}_{i} & =-k_{i} \sum_{j \in \mathcal{N}_{i}} \frac{\partial V_{i j}}{\partial \xi_{i}}\left(\hat{x}_{i}, \xi_{i}\right)^{\top}, \\
u_{i} & =-R_{j j}^{-1} \hat{g}_{i j}^{\top}\left(\hat{x}_{i}\right) \frac{\partial V_{i j}}{\partial \bar{x}_{i}}\left(\hat{x}_{i}, \xi_{i}\right)^{\top}
\end{aligned}
$$

for $i=1, \ldots, N$. Note that the dynamic control law (15) is equivalent to (14) computed with the actual local states $\hat{x}_{i}$ in place of $\bar{x}_{i}$. Let $x_{\mathcal{N}_{i}}^{-i} \triangleq\left[x_{j_{2}}^{\top}, \ldots, x_{j_{\mid \mathcal{N}_{i}}}^{\top}\right]^{\top}$, $j_{2}, \ldots, j_{\left|\mathcal{N}_{i}\right|} \in \mathcal{N}_{i} \backslash\{i\}$ denote the local state of agent $i$ excluding its own individual state $x_{i}$, and let $X_{i} \triangleq$ $\left[x_{i}^{\top}, \xi_{i}^{\top}\right]^{\top}$ denote the individual extended state. Finally, let $\hat{g}_{i j}^{\top} \triangleq\left[g_{i i}^{\top}\left(x_{i}\right), g_{i \mathcal{N}_{i}}^{\top}\left(x_{\mathcal{N}_{i}}^{-i}\right)\right]^{\top}$. The state of the closedloop system (2)-(15), i.e. $\left(x, \xi_{1}, \ldots, \xi_{N}\right)$, can be described as the collection of the individual extended states $X_{i}$, for $i=1, \ldots, N$, which satisfy dynamics

$$
\begin{aligned}
\dot{X}_{i}=\left(A_{i}\left(X_{i}\right)\right. & \left.+A_{\mathcal{N}_{i}}\left(X_{i}, x_{\mathcal{N}_{i}}^{-i}\right)\right) X_{i} \\
& +\sum_{j \in \mathcal{N}_{i}} B_{i j}\left(X_{i}, X_{j}, x_{\mathcal{N}_{j}}^{-j}\right)\left[\begin{array}{c}
X_{j} \\
x_{\mathcal{N}_{j}}^{-j}
\end{array}\right],
\end{aligned}
$$

with $A_{i}\left(X_{i}\right), A_{\mathcal{N}_{i}}\left(X_{i}, x_{\mathcal{N}_{i}}^{-i}\right)$ and $B_{i j}\left(X_{i}, X_{j}, x_{\mathcal{N}_{j}}^{-j}\right)$ defined (overleaf) in (17), for $j \in \mathcal{N}_{i}$, wherein $\Psi_{i j}: \mathbb{R}^{\hat{n}_{i}} \times \mathbb{R}^{\hat{n}_{i}} \rightarrow$ $\mathbb{R}^{\hat{n}_{i} \times \hat{n}_{i}}$ is the Jacobian of $\left(\frac{1}{2} P_{i j}\left(\xi_{i}\right) \bar{x}_{i}\right)$ with respect to $\xi_{i}$, and $\Psi\left(\bar{x}_{i}, \xi_{i}\right) \triangleq \bar{\Psi}\left(x_{i}, \xi_{i}\right)+\tilde{\Psi}\left(x_{\mathcal{N}_{i}}^{-i}, \xi_{i}\right)$, for $j \in \mathcal{N}_{i}$, for $i=1, \ldots, N$.

In the following statement, we provide sufficient conditions for asymptotic stability of the global system (2) in closed-loop with (15), for $i=1, \ldots, N$.

Theorem 2: Suppose Assumption 1 holds and consider the individual extended systems (16), for $i=$ $1, \ldots, N$. Let $\gamma_{i}: \mathbb{R}^{n_{i}+\hat{n}_{i}} \rightarrow \mathbb{R}$ and $\bar{V}_{i}: \mathbb{R}^{n_{i}+\hat{n}_{i}} \rightarrow \mathbb{R}, \bar{V}_{i} \in$ $\mathcal{C}^{1}$, for $i=1, \ldots, N$, denote positive definite functions. Suppose that there exist constants $\sigma_{i}>0, a_{i j} \in \mathbb{R}$, for $j \in \mathcal{N}_{i}$, continuous functions $\alpha_{i}: \mathbb{R}^{\hat{n}_{i}-n_{i}} \rightarrow \mathbb{R}$, and sets $\chi_{i} \subseteq \mathbb{R}^{n_{i}+\hat{n}_{i}}, 0 \in \chi_{i}$, and $\bar{\chi}_{i} \triangleq \chi_{1} \times \cdots \times \chi_{i}$, for $i=1, \ldots, N$, such that the conditions
(i) $\frac{\partial V_{i}}{\partial X_{i}} A_{i}\left(X_{i}\right) X_{i} \leq-\sigma_{i} \cdot \gamma_{i}\left(X_{i}\right)$
(ii) $\frac{\partial \bar{V}_{i}}{\partial X_{i}} A_{\mathcal{N}_{i}}\left(X_{i}, x_{\mathcal{N}_{i}}^{-i}\right) X_{i} \leq \alpha_{i}\left(x_{\mathcal{N}_{i}}^{-i}\right) \cdot \gamma_{i}\left(X_{i}\right)$
(iii) $\frac{\partial \bar{V}_{i}}{\partial X_{i}} \sum_{j \in \mathcal{N}_{i}} B_{j}\left(X_{i}, X_{j}, x_{\mathcal{N}_{j}}^{-j}\right)\left[\begin{array}{c}X_{j} \\ x_{\mathcal{N}_{j}}^{-j}\end{array}\right]$$$
\leq \gamma_{i}\left(X_{i}\right)^{\frac{1}{2}} \sum_{j=1}^{i-1} a_{i j} \gamma_{j}\left(X_{j}\right)^{\frac{1}{2}},
$$ 


$$
\begin{aligned}
& A_{i}\left(X_{i}\right) \triangleq\left[\begin{array}{cc}
F_{i}\left(x_{i}\right)-g_{i i}\left(x_{i}\right) R_{i i}^{-1} g_{i i}^{\top}\left(x_{i}\right)\left[I_{n_{i}} 0\right]\left(P_{i i}\left(\xi_{i}\right)+S_{i i}\right)\left[\begin{array}{c}
I_{n_{i}} \\
0
\end{array}\right] & g_{i i}\left(x_{i}\right) R_{i i}^{-1} g_{i i}^{\top}\left(x_{i}\right)\left[\begin{array}{ll}
I_{n_{i}} & 0
\end{array}\right] S_{i i} \\
-k_{i} \sum_{j \in \mathcal{N}_{i}}\left(\bar{\psi}_{i j}^{\top}\left(x_{i}, \xi_{i}\right)-S_{i j}\right)\left[\begin{array}{c}
I_{n_{i}} \\
0
\end{array}\right] & -k_{i} \sum_{j \in \mathcal{N}_{i}} S_{i j}
\end{array}\right] \\
& A_{\mathcal{N}_{i}}\left(X_{i}, x_{\mathcal{N}_{i}}^{-i}\right) \triangleq\left[\begin{array}{cc}
-g_{i i}\left(x_{i}\right) R_{i i}^{-1} g_{i \mathcal{N}_{i}}^{\top}\left(x_{\mathcal{N}_{i}}^{-i}\right)\left[\begin{array}{ll}
0 & I_{n_{i}}
\end{array}\right]\left(P_{i i}\left(\xi_{i}\right)+S_{i i}\right)\left[\begin{array}{c}
I_{n_{i}} \\
0
\end{array}\right] & g_{i i}\left(x_{i}\right) R_{i i}^{-1} g_{i \mathcal{N}_{i}}^{\top}\left(x_{\mathcal{N}_{i}}^{-i}\right)\left[\begin{array}{ll}
0 & I_{n_{i}}
\end{array}\right] S_{i i} \\
-k_{i} \sum_{j \in \mathcal{N}_{i}} \tilde{\psi}_{i j}^{\top}\left(x_{\mathcal{N}_{i}}^{-i}, \xi_{i}\right)\left[\begin{array}{c}
I_{n_{i}} \\
0
\end{array}\right] & 0
\end{array}\right] \\
& B_{i j} \triangleq\left[\begin{array}{cc}
-g_{i i} R_{i i}^{-1} \hat{g}_{i j}^{\top}\left(P_{i i}+S_{i i}\right)\left[\begin{array}{c}
0 \\
I_{n_{j}}
\end{array}\right]-g_{i j} R_{j j}^{-1} \hat{g}_{j j}^{\top}\left(P_{j j}+S_{j j}\right)\left[\begin{array}{c}
I_{n_{j}} \\
0
\end{array}\right] g_{i j} R_{j j}^{-1} \hat{g}_{j j}^{\top} S_{j j}-g_{i j} R_{j j}^{-1} \hat{g}_{j j}^{\top}\left(P_{j j}+S_{j j}\right)\left[\begin{array}{c}
0 \\
I_{\hat{n}_{j}-n_{j}}
\end{array}\right] \\
-k_{i} \sum_{j \in \mathcal{N}_{i}}\left(\psi_{i j}^{\top}\left(\hat{x}_{i}, \xi_{i}\right)-S_{i j}\right)\left[\begin{array}{c}
0 \\
I_{n_{j}} \\
0
\end{array}\right] & 0
\end{array}\right]
\end{aligned}
$$

with $\alpha_{i}\left(x_{\mathcal{N}_{i}}^{-i}\right)<\sigma_{i}$, for $i=1, \ldots, N$, hold for all $\left(X_{1}, \ldots, X_{N}\right) \in \bar{\chi}_{N}$. Then the origin of system (16), $i=1, \ldots, N$, is locally asymptotically stable.

Corollary 1: Suppose Assumption 1 holds and consider the linear autonomous system described by the dynamics $\dot{X}_{i}=\left(A_{i}(0)+A_{\mathcal{N}_{i}}(0,0)\right) X_{i}$. Suppose there exists a symmetric positive definite matrix $\tilde{P}_{i} \in$ $\mathbb{R}^{\left(n_{i}+\hat{n}_{i}\right) \times\left(n_{i}+\hat{n}_{i}\right)}$ such that

$$
\left.\tilde{P}_{i}\left(A_{i}+A_{\mathcal{N}_{i}}\right)\right|_{(0,0)}+\left.\left(A_{i}+A_{\mathcal{N}_{i}}\right)^{\top}\right|_{(0,0)} \tilde{P}_{i}<0,
$$

for $i=1, \ldots, N$. Then, the origin of system (16), for $i=1, \ldots, N$, is locally exponentially stable.

\section{Stabilising Solution}

The existence of a positive definite matrix $\tilde{P}_{i}$ such that condition (18) is satisfied, for $i=1, \ldots, N$, can be enforced in the solution of Problem 2, $i=1, \ldots, N$, by imposing an additional constraint to the conditions in Definition 4. This provides a solution to Problem 3, as detailed in the following statement.

Proposition 1: Consider system (1) and cost functionals (6), for $j \in \mathcal{N}_{i}$, subject to assumed dynamics (5), for $i=1, \ldots, N$. Suppose Assumptions 1 and 2, for $i=1, \ldots, N$, hold. Let $P_{i j}$, for $j \in \mathcal{N}_{i}$, be an algebraic $\bar{P}$ matrix solution of (7), for $j \in \mathcal{N}_{i}$, for which there exists a symmetric positive definite matrix $\tilde{P}_{i}$ such that (18) holds, for $i=1, \ldots, N$. Let $S_{i j} \in \mathbb{R}^{\hat{n}_{i} \times \hat{n}_{i}}$, $S_{i j}=S_{i j}^{\top}>0$, for $j \in \mathcal{N}_{i}$, be such that (12) is satisfied, for $i=1, \ldots, N$. Then, there exist $\bar{k}_{i} \geq 0$ and a set $\Omega_{i} \subseteq \mathbb{R}^{\hat{n}_{i}} \times \mathbb{R}^{\hat{n}_{i}}$, with $(0,0) \in \Omega_{i}$, such that the set of assumed admissible dynamic feedback strategies (14), for $j \in \mathcal{N}_{i}$, constitutes a feedback $\epsilon_{\alpha}$-Nash equilibrium of the $i$-th local differential game, for $i=1, \ldots, N$. Moreover the origin of (2)-(15), is locally asymptotically stable.

\section{EXAMPLE}

As an example of the problem formulation and of the control design methodology presented in this paper, we consider a MAS composed of three mobile agents satisfying single integrator dynamics

$$
\dot{p}_{i}=u_{i}, \quad i=1,2,3
$$

where $p_{i} \in \mathbb{R}^{3}$ represents the position coordinates of agent $i$ in a common inertial frame of reference. Let the relative-distance vectors $d_{10}, d_{21}$ and $d_{32}$ describe a desired formation, where $d_{i j}$ represents the desired distance vector from the position of agent $j$ to the position of agent $i$, for $i=1,2,3$, for $j=1,2,3$, and $d_{10}$ represents the distance of the position of agent 1 from the origin of the inertial frame of reference. We define error coordinates $x_{1} \triangleq p_{1}-d_{10}, x_{2} \triangleq p_{2}-p_{1}-d_{21}$, and $x_{3} \triangleq p_{3}-p_{2}-d_{32}$, which evolve according to dynamics

$$
\dot{x}_{1}=u_{1}, \quad \dot{x}_{2}=u_{2}-u_{1}, \quad \dot{x}_{3}=u_{3}-u_{2} .
$$

Note that the desired formation is achieved when $x_{i}=$ 0 , for $i=1,2,3$. Let the graph $\mathcal{G}(\mathcal{V}, \mathcal{E})$, with $\mathcal{V}=\{1,2,3\}$ and $\mathcal{E}=\{(1,2),(2,3)\}$, describe the communication between the agents. The sets of 1-hop neighbours are then $\mathcal{N}_{1}=\{1\}, \mathcal{N}_{2}=\{1,2\}, \mathcal{N}_{3}=\{2,3\}$, and the sets of 2-hop neighbours are $\mathcal{N}_{1}^{2}=\mathcal{N}_{2}^{2}=\emptyset$, and $\mathcal{N}_{3}^{2}=\{1\}$. The local states of agents 1,2 and 3 are $\hat{x}_{1}=x_{1}$, $\hat{x}_{2} \triangleq\left[x_{2}^{\top}, x_{1}^{\top}\right]^{\top}$ and $\hat{x}_{3} \triangleq\left[x_{3}^{\top}, x_{2}^{\top}\right]^{\top}$, respectively.

In addition to keeping a formation, each agent $i$ seeks to avoid collisions with agents $j \in \mathcal{N}_{i} \backslash\{i\}$. Therefore, "barrier functions" (as in [23]) are included in the running costs $q_{i j}$, for $j=2,3$. Using the barrier functions $b_{2}\left(x_{2}\right)=\left(\left\|x_{2}+d_{21}\right\|^{2}-r^{2}\right)^{-2}$ and $b_{3}\left(x_{3}, x_{2}\right)=$ $\left(\left\|x_{3}+d_{32}\right\|^{2}-r^{2}\right)^{-2}+\left(\left\|x_{3}+x_{2}+d_{32}+d_{21}\right\|^{2}-r^{2}\right)^{-2}$ for agents 2 and 3, respectively, where $r=1$ represents the minimum admissible distance between the agents, we consider the cost functionals (6), for $j \in \mathcal{N}_{i}, i=$ $1,2,3$, defined by $Q_{11}=I, Q_{21}=\operatorname{blockdiag}(0, I), Q_{22}=$ $\operatorname{blockdiag}\left(\left(1+b_{2}\left(x_{2}\right)\right) \cdot I, 0\right), Q_{32}=\operatorname{blockdiag}(0,(1+$ $\left.\left.b_{2}\left(x_{2}\right)\right) \cdot I\right), Q_{33}=\operatorname{blockdiag}\left(\left(1+b_{3}\left(x_{3}, x_{2}\right)\right) \cdot I, 0\right)$, and by $R_{i i}=I$, for $i=1,2,3$.

The local differential game corresponding to agent 1 is essentially a linear quadratic optimal control problem 


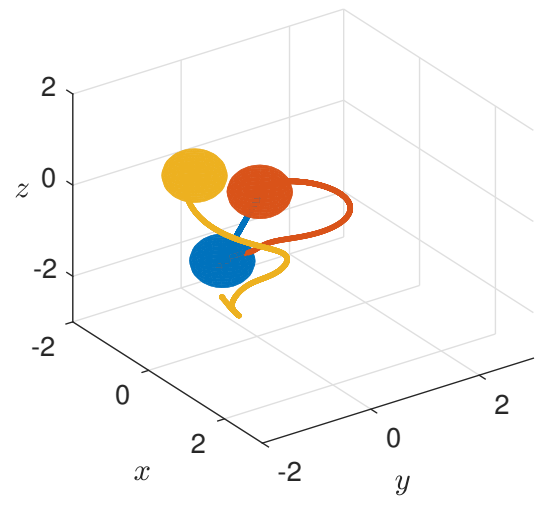

Fig. 1: The trajectories of $p_{1}$ (blue), $p_{2}$ (red) and $p_{3}$ (yellow $)^{4}$. Final positions are indicated by circular markers.

defined by the cost functional (6), for $j=i=1$, for which a stabilising solution is readily available (without the necessity of using a dynamic extension), i.e. agent 1 solves Problem 1. Agents 2 and 3, on the other hand, seek a solution of Problem 2 defined by cost functionals (6), for $j \in \mathcal{N}_{i}$, subject to the assumed local dynamics $\dot{\bar{x}}_{i}=\left[\left(u_{i}-\bar{u}_{i j}\right)^{\top}, \bar{u}_{i j}^{\top}\right]^{\top}$, for $j \in \mathcal{N}_{i}$, for $i=2,3$, respectively. The collection of all three local differential games constitutes the distributed differential game, namely Problem 3. Agent 1 adopts the control law $u_{1}=-x_{1}$, i.e. the solution of its local differential game. $P_{22}=\operatorname{blockdiag}\left(I\left(2+\sqrt{1+b_{2}\left(x_{2}\right)}\right), 2 I\right)$ and $P_{21}=\operatorname{blockdiag}(2 I, 3 I)$, and $P_{33}=\operatorname{blockdiag}(I(2+$ $\left.\left.\sqrt{1+b_{3}\left(x_{3}, x_{2}\right)}\right), 2 I\right)$ and $P_{32}=\operatorname{blockdiag}(2 I, 3 I)$, constitute algebraic $\bar{P}$ matrix solutions for the local differential games of agents 2 and 3, respectively. Agents 2 and 3 adopt the corresponding dynamic control laws (15), with $V_{i j}$ defined by (13), for $j \in \mathcal{N}_{i}$, for $i=2,3$. The remaining parameters are defined as $k_{i}=10$ and $S_{i j}=30 I$, for $j \in \mathcal{N}_{i}$ for $i=2,3$. This set of control laws (for agents 1,2 and 3) constitutes a solution to Problem 3 and is such that the origin of the closedloop system (20) is locally asymptotically stable (as implied by Corollary 1). A simulation of the resulting closed-loop system has been run for the formation defined by $d_{10}=[-1,0,-2]^{\top}, d_{21}=[1,0,2]^{\top}$ and $d_{32}=[-1,-0.5,0]^{\top}$, with initial conditions $p_{1}(0)=$ $[0,0,0]^{\top}, p_{2}(0)=[-1,0,-2]^{\top}, p_{3}(0)=[0.5,-1,-1.5]^{\top}$, $\xi_{2}(0)=[1,1,0,1,1,0]^{\top}$ and $\xi_{3}(0)=[10,-2,0,4,0,0]^{\top}$. The collision-free trajectories of $p_{1}$ (blue), $p_{2}$ (red) and $p_{3}$ (yellow) are shown in Figure 1.

\section{CONCLUSION}

The distributed control of a class of MAS is considered in this paper. A theoretical framework based on the solution of local differential games subject to additional stability conditions has been given, providing a

\footnotetext{
${ }^{4}$ For a video representation see https://drive.google.com/ open?id=1m1h_MfQrZ6h0rw3a2kSDrmo5AKolaTLV
}

systematic way to design distributed control laws for MAS with an acyclic communication topology.

\section{REFERENCES}

[1] M. Mesbahi and M. Egerstedt, Graph Theoretic Methods in Multiagent Networks, 1st ed. Princeton University Press, 2010.

[2] J. S. Shamma, Cooperative Control of Distributed Multi-Agent Systems, 1st ed. Wiley, 2007.

[3] T. Arai, E. Pagello, and L. E. Parker, "Editorial: Advances in multi-robot systems," IEEE Transactions on Robotics and Automation, vol. 18, no. 5, pp. 655-661, 2002.

[4] R. Olfati-Saber, "Flocking for multi-agent dynamic systems: algorithms and theory," IEEE Transactions on Automatic Control, vol. 51, no. 3, pp. 401-420, 2006.

[5] D. Cappello, S. Garcin, Z. Mao, M. Sassano, A. Paranjape, and T. Mylvaganam, "A hybrid controller for multi-agent collision avoidance via a differential game formulation," IEEE Transactions on Control Systems Technology, (under review).

[6] R. D'Andrea, T. Kalmár-Nagy, P. Ganguly, and M. Babish, "The cornell robocup team," in RoboCup 2000: Robot Soccer World Cup IV. Springer-Verlag, 2001.

[7] W. Lin, "Distributed UAV formation control using differential game approach," Aerospace Science and Technology, vol. 35, pp. $54-62,2014$.

[8] C.-K. Tham and J. Renaud, "Multi-agent systems on sensor networks: A distributed reinforcement learning approach," 2005 International Conference on Intelligent Sensors, Sensor Networks and Information Processing, 2005.

[9] T. Mylvaganam and A. Astolfi, "Control of microgrids using a differential game theoretic framework," in 54th IEEE Conference on Decision and Control, 2015, pp. 5839-5844.

[10] N. C. Ekneligoda and W. W. Weaver, "Optimal transient control of microgrids using a game theoretic approach," 2011 IEEE Energy Conversion Congress and Exposition, 2011.

[11] A. Rantzer, "Using game theory for distributed control engineering," Department of Automatic Control, Lund Institute of Technology, Lund University, Tech. Rep., 2008.

[12] R. Olfati-Saber and R. M. Murray, "Distributed cooperative control of multiple vehicle formations using structural potential functions," IFAC Proceedings Volumes, vol. 35, no. 1, pp. 495-500, 2002.

[13] D. Siljak, Decentralized Control of Complex Systems. Academic Press, 1991.

[14] L. Bakule, "Decentralized control: An overview," Annual Reviews in Control, vol. 32, no. 1, pp. 87 - 98, 2008.

[15] W. Lin, "Differential games for multi-agent systems under distributed information," Electronic Theses and Dissertations, University of Central Florida, 2013.

[16] F. Fele, E. Debada, J. M. Maestre, and E. F. Camacho, "Coalitional control for self-organizing agents," IEEE Transactions on Automatic Control, vol. 63, 2018.

[17] T. Mylvaganam and A. Astolfi, "Towards a systematic solution for differential games with limited communication," in American Control Conference, 2016, pp. 3814-3819.

[18] T. Mylvaganam, "A game theoretic approach to distributed control of homogeneous multi-agent systems," in The 56th IEEE Conference on Decision and Control, 2017, pp. 6217-6222.

[19] D. Cappello and T. Mylvaganam, "Distributed control of multiagent systems via linear quadratic differential games with partial information," in 57th IEEE Conference on Decision and Control, 2019.

[20] K. G. Vamvoudakis, F. L. Lewis, and G. R. Hudas, "Multi-agent differential graphical games: Online adaptive learning solution for synchronization with optimality," Automatica, no. 48, pp. 1598-1611, 2012.

[21] T. Basar and G. Olsder, Dynamic Noncooperative Game Theory. Academic Press, 1982.

[22] T. Mylvaganam, M. Sassano, and A. Astolfi, "Constructive $\epsilon$-Nash equilibria for nonzero-sum differential games," IEEE Transactions on Automatic Control, vol. 60, no. 4, pp. 950-965, 2015.

[23] T. Mylvaganam and M. Sassano, "Autonomous collision avoidance for wheeled mobile robots using a differential game approach," European Journal of Control, vol. 40, pp. 53-61, 2018. 